\title{
Four-neutrino oscillations and the solar neutrino problem
}

\author{
C. Giunti ${ }^{1}$, M.C. Gonzalez-Garcia ${ }^{2}$ and C. Peña-Garay ${ }^{2}$ \\ ${ }^{1}$ INFN, Sez. di Torino, and Dip. di Fisica Teorica, Univ. di Torino, I-10125 Torino, Italy \\ 2 Instituto de Física Corpuscular (IFIC) CSIC-Universidad de Valencia, \\ Edificio Institutos de Paterna, Apartado 2085, 46071 Valencia
}

(July 14, 2000)

\begin{abstract}
We perform a fit of solar neutrino data in the framework of the two four-neutrino schemes that are compatible with the results of all neutrino oscillation experiments. These schemes allow simultaneous transitions of solar $\nu_{e}$ 's into active $\nu_{\mu}$ 's, $\nu_{\tau}$ 's and sterile $\nu_{s}$. The data imply that the SMA solution is valid for any combination of $\nu_{e} \rightarrow$ active and $\nu_{e} \rightarrow$ sterile transitions, whereas the LMA, LOW and VO solutions disappear when $\nu_{e} \rightarrow \nu_{s}$ transitions are dominant.

DFTT 29/00, arXiv:hep-ph/0007154.

Talk presented by C. Giunti at the NuFact'00 Workshop, May 22-26, 2000, Naval Postgraduate School, Monterey, California, USA.
\end{abstract}

\section{INTRODUCTION: WHY FOUR-NEUTRINO MIXING?}

Evidences in favor of neutrino oscillations (see [1]) have been found in solar (Homestake [2], Kamiokande [3], GALLEX [4], SAGE [5], Super-Kamiokande [6]) and atmospheric (Kamiokande, IMB, Super-Kamiokande, Soudan 2, MACRO 7) neutrino experiments and in the LSND experiment [8]. It is rather well-known [9. 10] that these three evidences require the existence of at least three small neutrino mass-squared differences $\left(\Delta m^{2}\right.$ 's):

$$
\begin{gathered}
\Delta m_{\mathrm{sun}}^{2} \sim 10^{-10}-10^{-4} \mathrm{eV}^{2}, \\
\Delta m_{\mathrm{atm}}^{2} \sim 10^{-3}-10^{-2} \mathrm{eV}^{2}, \\
\Delta m_{\mathrm{LSND}}^{2} \sim 10^{-1}-10 \mathrm{eV}^{2} .
\end{gathered}
$$

This means that at least four light massive neutrinos must exist in nature. Following the old principle known as Occam's razor ("pluralitas non est ponenda sine necessitate") we assume the existence of four light massive neutrinos, that is the minimal possibility compatible with all data. In this case the left-handed components $\nu_{\alpha L}$ of the flavor neutrino fields are superpositions of the lefthanded components $\nu_{k L}(k=1, \ldots, 4)$ of neutrino fields with definite mass $m_{k}$ smaller than a few $\mathrm{eV}$ :

$$
\nu_{\alpha L}=\sum_{k=1}^{4} U_{\alpha k} \nu_{k L},
$$

where $U$ is a $4 \times 4$ unitary mixing matrix.

From the measurement of the invisible decay width of the $Z$-boson it is known that the number of light active neutrino flavors is three (see [11]), corresponding to $\nu_{e}$, $\nu_{\mu}$ and $\nu_{\tau}$ (active neutrinos are those taking part to standard weak interactions). This implies that the number of massive neutrinos is bigger or equal to three. If there are four massive neutrinos, in the flavor basis there is one sterile neutrino, $\nu_{s}$, that does not take part to standard weak interactions. For the flavor index $\alpha$ in Eq. (ד) we choose the ordering $\alpha=e, s, \mu, \tau$.
Let us emphasize that four-neutrino mixing is very interesting both for theory and experiment. From the theoretical point of view the existence of a light sterile neutrino can be explained only with models far beyond the Standard Model and is thus a signal of exciting new physics. From the experimental point of view four-neutrino mixing is attractive because it allows the existence of a $\Delta m^{2}$ at the $\mathrm{eV}$ scale which generates oscillations in different channels that can be explored with high precision in short-baseline experiments. Moreover, it allows large CP-violation effects in long-baseline and $\nu$-factory experiments (see [12]). Four-neutrino mixing may also open the possibility for measurable exotic phenomena, as neutrino decay, neutrino magnetic moment, etc.

From the model-builder point of view, there seems to be no difficulty in constructing models with a light sterile neutrino (as one can convince oneself with an appropriate search of the hep-ph electronic archive, resulting in too many four-neutrino models to be cited here). However, our impression is that these models are constructed with assumptions "ad hoc" in order to generate a light sterile neutrino, with the possible exception of models based on the existence of a mirror world (see 13]) and models based on the existence of large extra dimensions (see [14]), in which light neutral fermions that mix with the ordinary neutrinos seem to be naturally allowed.

Our approach is phenomenological: we extract information on four-neutrino mixing (masses and mixing angles) from the available data.

\section{ALLOWED FOUR-NEUTRINO SCHEMES}

The six types of four-neutrino mass spectra with three different scales of $\Delta m^{2}$ that can accommodate the hierarchy $\Delta m_{\text {sun }}^{2} \ll \Delta m_{\text {atm }}^{2} \ll \Delta m_{\mathrm{LSND}}^{2}$ (see Eqs. (1)-(3)) are shown qualitatively in Fig. 1. In all these mass spectra there are two groups of close masses separated by the "LSND gap" of the order of $1 \mathrm{eV}$. The six schemes 


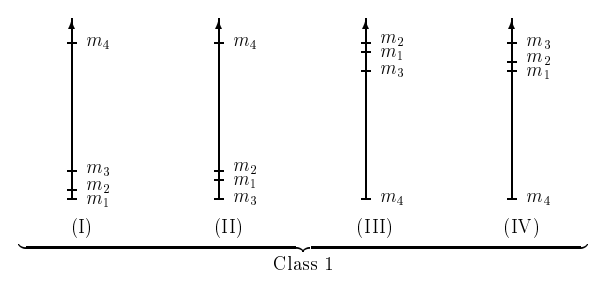

Figure 1

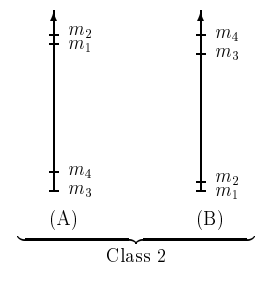

are divided into four schemes of class 1 (I-IV) in which there is a group of three masses separated from an isolated mass by the LSND gap, and two schemes of class $2(\mathrm{~A}, \mathrm{~B})$ in which there are two couples of close masses separated by the LSND gap. In each scheme the smallest mass-squared difference $\left(\Delta m_{i j}^{2} \equiv m_{i}^{2}-m_{j}^{2}\right)$ corresponds to $\Delta m_{21}^{2}=\Delta m_{\text {sun }}^{2}$, the intermediate one to $\Delta m_{\mathrm{atm}}^{2}$ $\left(\Delta m_{31}^{2} \simeq \Delta m_{32}^{2}\right.$ in the schemes of class $1, \Delta m_{43}^{2}$ in the schemes of class 2) and the largest mass squared difference $\Delta m_{41}^{2}=\Delta m_{\mathrm{LSND}}^{2}$ is relevant for the oscillations observed in the LSND experiment $\left(\Delta m_{41}^{2} \simeq \Delta m_{42}^{2} \simeq \Delta m_{43}^{2}\right.$ in the schemes of class $1, \Delta m_{41}^{2} \simeq \Delta m_{42}^{2} \simeq \Delta m_{31}^{2} \simeq$ $\Delta m_{32}^{2}$ in the schemes of class 2).

It has been show that the four schemes of class 1 are disfavored by the data if also the negative results of shortbaseline accelerator and reactor disappearance neutrino oscillation experiments are taken into account 15 17.97. The reason is simple: in order to explain solar and atmospheric data with neutrino oscillations $\nu_{e}$ and $\nu_{\mu}$ must have large mixing with the group of three neutrinos and, consequently, small mixing with the isolated neutrino. In this case, short-baseline oscillations occurring through the mass-squared difference $\Delta m_{41}^{2}$, i.e. through the interference of the wave functions of the isolated neutrino and the three grouped neutrinos, are strongly suppressed. It turns out that the negative results of short-baseline accelerator and reactor disappearance experiments imply that the amplitude of short-baseline $\nu_{\mu} \rightarrow \nu_{e}$ transitions is suppressed below the value measured by LSND [15, 17, 2, Hence, the schemes of class 1 cannot explain all neutrino oscillation data.

On the other hand, the two four-neutrino schemes of class 2 are compatible with the results of all neutrino oscillation experiments if the mixing of $\nu_{e}$ with $\nu_{1}$ and $\nu_{2}$, the two mass eigenstates responsible for the oscillations of solar neutrinos, and the mixing of $\nu_{\mu}$ with $\nu_{3}$ and $\nu_{4}$, the two mass eigenstates responsible for the oscillations of atmospheric neutrinos, are large 15 17,95. Hence, we have the two possibilities illustrated in Fig. 2, in which $\nu_{e}$ and $\nu_{\mu}$ are depicted close to the two massive neutrinos with which they have large mixing and it is explicitly indicated that the mixing of $\nu_{\tau}$ and $\nu_{s}$ is unknown. Information on the mixing of $\nu_{\tau}$ and $\nu_{s}$, as well as more detailed information on the mixing of $\nu_{e}$ and $\nu_{\mu}$, can be obtained through the fit of solar and atmospheric neu-

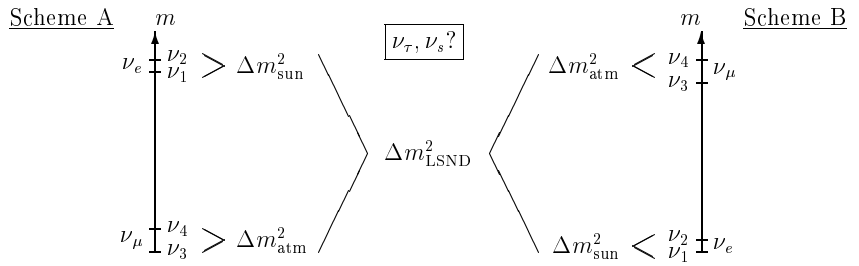

Figure 2

trino data, and, in the future, of the data of long-baseline experiments.

The appropriate formalism for the treatment of solar and atmospheric neutrino oscillations, including matter effects, in the framework of the two four-neutrino schemes $\mathrm{A}$ and $\mathrm{B}$ has been presented in Ref. [18]. In the next section we present the results of a fit of solar neutrino data 19].

\section{FIT OF SOLAR NEUTRINO DATA}

In the framework of the two four-neutrino schemes A and $\mathrm{B}$, solar neutrino oscillations depend on only one mass-squared difference, $\Delta m_{\text {sun }}^{2}$, but there are several degrees of freedom coming from the $4 \times 4$ mixing matrix, that can be parameterized in terms of six mixing angles and three $\mathrm{CP}$-violating phases (three additional $\mathrm{CP}$-violating phases are possible if massive neutrinos are Majorana particles, but can be neglected in the present contest because they do not have any effect in neutrino oscillations). However, the data of solar neutrino experiments together with those of short-baseline $\bar{\nu}_{e}$ disappearance experiments indicate that the elements $U_{e 3}$ and $U_{e 4}$ of the neutrino mixing matrix are very small 15.20]. This implies that two mixing angles are small and can be neglected in the study of solar (and atmospheric) neutrino oscillations [18]. Furthermore, the CP-violating phases can be neglected because their effects are washed out by the average over neutrino energy and source-detector distance. Therefore, we consider the mixing matrix $U$ in Fig. 3 [18, 19, where $\vartheta_{12}, \vartheta_{23}, \vartheta_{24}, \vartheta_{34}$ are four mixing angles and $c_{i j} \equiv \cos \vartheta_{i j}$ and $s_{i j} \equiv \sin \vartheta_{i j}$. The rows and columns of the mixing matrix $U$ correspond, respectively, to the neutrino flavors $\nu_{e}, \nu_{s}, \nu_{\mu}, \nu_{\tau}$ and to the mass eigenstates $\nu_{1}, \nu_{2}, \nu_{3}, \nu_{4}$.

Since solar neutrino oscillations are generated by the mass-square difference between $\nu_{2}$ and $\nu_{1}$ and $U_{e 1}=$ $\cos \vartheta_{12}, U_{e 2}=\sin \vartheta_{12}$, the survival of solar $\nu_{e}$ 's, $P_{\nu_{e} \rightarrow \nu_{e}}$, mainly depends on the mixing angle $\vartheta_{12}$, with the only correction due to four-neutrino mixing in the matter potential that is given by

$$
V \equiv V_{C C}+\cos ^{2} \vartheta_{23} \cos ^{2} \vartheta_{24} V_{N C}
$$

where $V_{C C}=\sqrt{2} G_{F} N_{e}$ and $V_{N C}=-\sqrt{2} G_{F} N_{n} / 2$ are 


$$
U=\left(\begin{array}{cccc}
c_{12} & s_{12} & 0 & 0 \\
-s_{12} c_{23} c_{24} & c_{12} c_{23} c_{24} & s_{23} c_{24} & s_{24} \\
s_{12}\left(c_{23} s_{24} s_{34}+s_{23} c_{34}\right) & -c_{12}\left(s_{23} c_{34}+c_{23} s_{24} s_{34}\right) & c_{23} c_{34}-s_{23} s_{24} s_{34} & c_{24} s_{34} \\
s_{12}\left(c_{23} s_{24} c_{34}-s_{23} s_{34}\right) & c_{12}\left(s_{23} s_{34}-c_{23} s_{24} c_{34}\right) & -\left(c_{23} s_{34}+s_{23} s_{24} c_{34}\right) & c_{24} c_{34}
\end{array}\right)
$$

Figure 3

the usual charged-current and neutral-current potentials (see [1]).

The mixing angles $\vartheta_{23}$ and $\vartheta_{24}$ determine the relative amount of transitions into sterile $\nu_{s}$ or active $\nu_{\mu}$ and $\nu_{\tau}$ (that cannot be distinguished in solar neutrino experiments, because their matter potential and their interaction in the detectors are equal, due only to neutralcurrent weak interactions). The active/sterile ratio and solar neutrino oscillations in general do not depend on the mixing angle $\vartheta_{34}$, that contribute only to the different mixings of $\nu_{\mu}$ and $\nu_{\tau}$. Indeed, the mixing of $\nu_{s}$ with $\nu_{1}$ and $\nu_{2}$ depends only on $\vartheta_{12}$ and the product $\cos \vartheta_{23} \cos \vartheta_{24}$. Moreover, instead of $\nu_{\mu}$ and $\nu_{\tau}$, one can consider the linear combinations

$$
\left(\begin{array}{c}
\nu_{a} \\
\nu_{b}
\end{array}\right)=\left(\begin{array}{cc}
-\sin \vartheta & -\cos \vartheta \\
\cos \vartheta & -\sin \vartheta
\end{array}\right)\left(\begin{array}{cc}
s_{34} & c_{34} \\
c_{34} & -s_{34}
\end{array}\right)\left(\begin{array}{c}
\nu_{\mu} \\
\nu_{\tau}
\end{array}\right),
$$

with $\tan \vartheta=\sin \vartheta_{24} / \tan \vartheta_{23}$. The mixing of $\nu_{a}$ and $\nu_{b}$ with $\nu_{1}$ and $\nu_{2}$ is given by $U_{a 1}=-s_{12} \sqrt{1-c_{23}^{2} c_{24}^{2}}, U_{a 2}=$ $c_{12} \sqrt{1-c_{23}^{2} c_{24}^{2}}, U_{b 1}=U_{b 2}=0$. Therefore, the oscillations of solar neutrinos depend only on $\vartheta_{12}$ and the product $\cos \vartheta_{23} \cos \vartheta_{24}$. If $0<\cos \vartheta_{23} \cos \vartheta_{24}<1$, solar $\nu_{e}$ 's can transform simultaneously into sterile neutrinos and in the linear combination $\nu_{a}$ of active $\nu_{\mu}$ and $\nu_{\tau}$, with the limiting cases of pure two-generation $\nu_{e} \rightarrow \nu_{a}$ transitions if $\cos \vartheta_{23} \cos \vartheta_{24}=0\left(U_{s 1}=U_{s 2}=0, U_{a 1}=-\sin \vartheta_{12}\right.$, $\left.U_{a 2}=\cos \vartheta_{12}\right)$, and pure two-generation $\nu_{e} \rightarrow \nu_{s}$ transitions if $\cos \vartheta_{23} \cos \vartheta_{24}=1\left(U_{s 1}=-\sin \vartheta_{12}, U_{s 2}=\cos \vartheta_{12}\right.$ and $U_{a 1}=U_{a 2}=0$ ).

Since the mixing of $\nu_{e}$ with $\nu_{1}$ and $\nu_{2}$ is equal to the one in the case of two-generations (with the mixing angle $\left.\vartheta_{12}\right)$, the mixing of $\nu_{s}$ with $\nu_{1}$ and $\nu_{2}$ is equal to the one in the case of two-generations times $\cos \vartheta_{23} \cos \vartheta_{24}$ and the mixing of $\nu_{a}$ with $\nu_{1}$ and $\nu_{2}$ is equal to the one in the case of two-generations times $\sqrt{1-\cos ^{2} \vartheta_{23} \cos ^{2} \vartheta_{24}}$, it is clear that in the general case of simultaneous $\nu_{e} \rightarrow \nu_{s}$ and $\nu_{e} \rightarrow \nu_{a}$ oscillations the corresponding transition probabilities are given by

$$
\begin{aligned}
& P_{\nu_{e} \rightarrow \nu_{s}}=\cos ^{2} \vartheta_{23} \cos ^{2} \vartheta_{24}\left(1-P_{\nu_{e} \rightarrow \nu_{e}}\right) \\
& P_{\nu_{e} \rightarrow \nu_{a}}=\left(1-\cos ^{2} \vartheta_{23} \cos ^{2} \vartheta_{24}\right)\left(1-P_{\nu_{e} \rightarrow \nu_{e}}\right)
\end{aligned}
$$

These expressions satisfy the relation of probability conservation $P_{\nu_{e} \rightarrow \nu_{e}}+P_{\nu_{e} \rightarrow \nu_{s}}+P_{\nu_{e} \rightarrow \nu_{a}}=1$. We cal-

\begin{tabular}{|c|c|c|c|}
\hline Experiment & Rate & Units & BP98 \\
\hline Homestake & $2.56 \pm 0.23$ & SNU & $\overline{7.8 \pm 1.1}$ \\
\hline GALLEX+SAGE & $72.3 \pm 5.6$ [象, & $\mathrm{SNU}$ & $130 \pm 7$ \\
\hline Kamiokande & $2.80 \pm 0.38[3]$ & $10^{6} \mathrm{~cm}^{-2} \mathrm{~s}^{-1}$ & $5.2 \pm 0.9$ \\
\hline Super-Kamiokande & $2.45 \pm 0.08$ & $10^{6} \mathrm{~cm}^{-2} \mathrm{~s}^{-1}$ & $5.2 \pm 0.9$ \\
\hline
\end{tabular}
culated the survival probability $P_{\nu_{e} \rightarrow \nu_{e}}$ in the range $10^{-11} \mathrm{eV}^{2} \leq \Delta m^{2} \leq 10^{-13} \mathrm{eV}^{2}, 10^{-4} \leq \tan ^{2} \vartheta_{12} \leq 10$ and $0 \leq \cos ^{2} \vartheta_{23} \cos ^{2} \vartheta_{24} \leq 1$, taking into account matter
Table

effects for $10^{-8} \mathrm{eV}^{2} \leq \Delta m_{21}^{2} \leq 10^{-3} \mathrm{eV}^{2}$ and the possible regeneration of $\nu_{e}$ 's when the flux of solar neutrinos crosses the Earth (for details see Ref. [19]).

In order to calculate the allowed regions for the parameters $\Delta m_{21}^{2}, \tan ^{2} \vartheta_{12}$ and $c_{23}^{2} c_{24}^{2}$, we have used data on the total event rates measured in the Chlorine experiment at Homestake [2], in the two Gallium experiments GALLEX [4] and SAGE [5] and in the water Cherenkov detectors Kamiokande [3] and Super-Kamiokande [6] shown in Table I. We used also the zenith angle distribution of the events and the electron recoil energy spectrum measured in the Super-Kamiokande experiment (825-day data sample) [6]. For the calculation of the theoretical expectations we use the BP98 standard solar model of Ref. [21]. The calculation of the theoretical rates, the statistical treatment of the data and the results of different fits of rates only, rates and zenith angle distribution, rates and recoil electron energy spectrum are described in Ref. [19]. Here we present only the result of the global $\chi^{2}$ fit of all data.

Figure shows the allowed regions at $90 \%$ and $99 \% \mathrm{CL}$ in the $\tan ^{2} \vartheta_{12}-\Delta m_{21}^{2}$ plane for $c_{23}^{2} c_{24}^{2}=$ $0,0.2,0.4,0.5,0.71$, that we calculated in the following way. We computed for a grid of points in the threedimensional $\tan ^{2} \vartheta_{12}-\Delta m_{21}^{2}-c_{23}^{2} c_{24}^{2}$ parameter space the expected values of the observables and with those and the corresponding uncertainties we constructed the function $\chi^{2}\left(\tan ^{2} \vartheta_{12}, \Delta m_{12}^{2}, c_{23}^{2} c_{24}^{2}\right)$. We found its minimum, $\chi_{\min }^{2}$, in the full three-dimensional space considering as a unique framework both MSW and vacuum oscillations. The allowed three-dimensional regions for a given CL are then defined as the set of points satisfying the condition

$$
\chi^{2}\left(\tan ^{2} \vartheta_{12}, \Delta m_{12}^{2}, c_{23}^{2} c_{24}^{2}\right)-\chi_{\min }^{2} \leq \Delta \chi^{2}(\mathrm{CL}, 3 \text { dof })
$$

where $\Delta \chi^{2}(\mathrm{CL}, 3$ dof $)=6.25,11.36$ for $\mathrm{CL}=90 \%, 99 \%$, respectively. In Fig. A we plotted the sections of such volumes in the $\tan ^{2} \vartheta_{12}-\Delta m_{21}^{2}$ plane for six selected values of $c_{23}^{2} c_{24}^{2}$.

The global minimum of $\chi^{2}, \chi_{\min }^{2}=28.8$ with 23 degrees of freedom corresponding to 28 data points (4 rates plus 6 Super-Kamiokande zenith-angle bins plus 18 Super-Kamiokande energy spectrum bins) minus 2 normalization factors (for the Super-Kamiokande zenithangle and energy spectrum data) minus 3 fitted parameters, lies in the LMA region for $\cos ^{2} \vartheta_{23} \cos ^{2} \vartheta_{24}=0$, corresponding to pure two-generation $\nu_{e} \rightarrow \nu_{a}$ oscillations, and $\tan ^{2} \vartheta_{12}=0.35, \Delta m_{21}^{2}=2.3 \times 10^{-5} \mathrm{eV}^{2}$. 


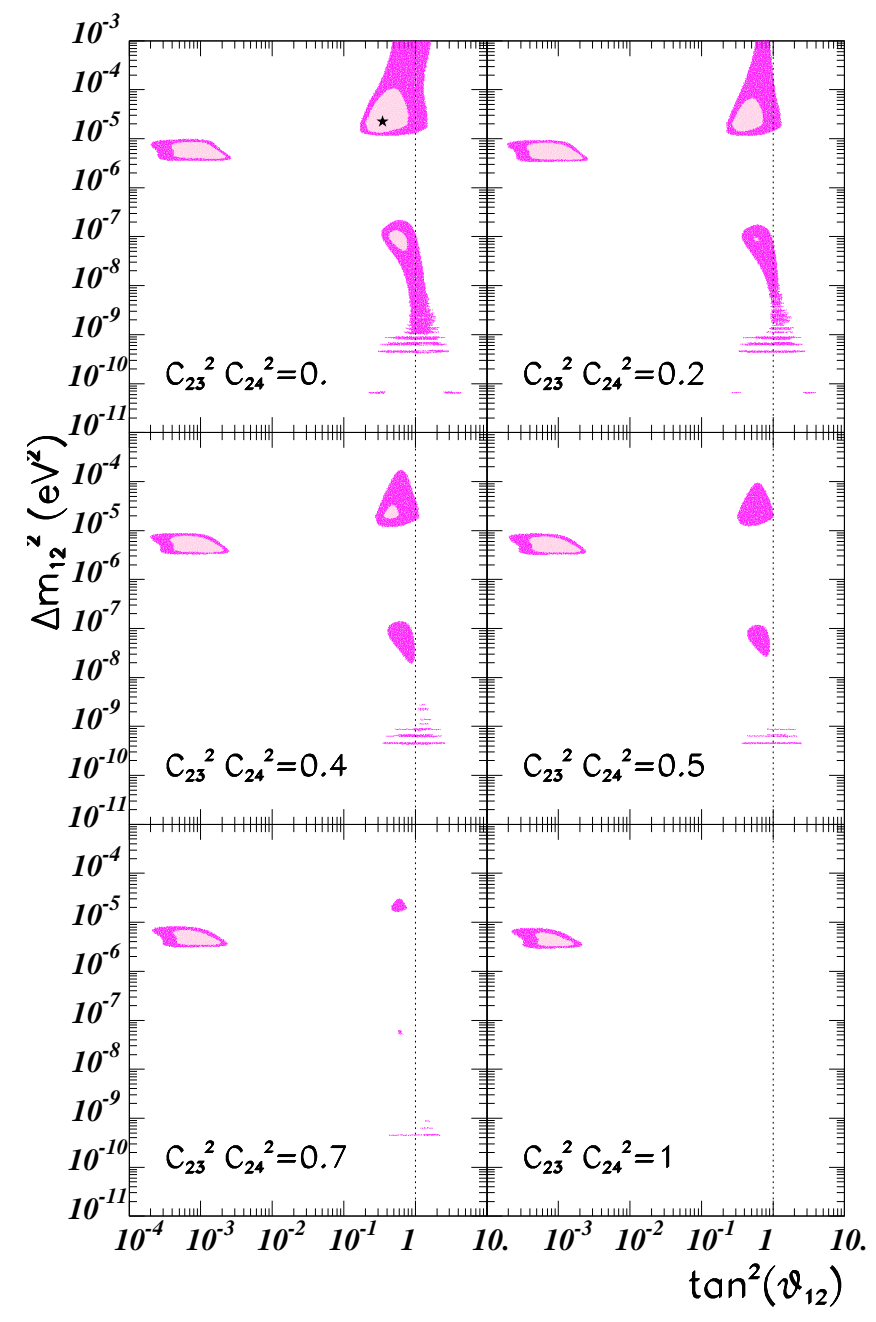

Figure 目

However, as one can see from Fig 5, the $\chi^{2}$ of the best-fit point in the LMA, LOW and VO regions rises steeply when $\cos ^{2} \vartheta_{23} \cos ^{2} \vartheta_{24}$ is increased. Therefore, the $99 \%$ CL LMA, LOW and VO regions disappear for $c_{23}^{2} c_{24}^{2} \simeq 0.78,0.70,0.83$, respectively. For values of $c_{23}^{2} c_{24}^{2}$ close to one, corresponding to almost pure twogenerations $\nu_{e} \rightarrow \nu_{s}$ transitions, only the SMA region is allowed. This is expected, because only the SMA solution is allowed in the two-generation $\nu_{e} \rightarrow \nu_{s}$ fit of solar data (see 221). Notice, however, that the statistical analysis is different: in the two-generation picture the pure $\nu_{e} \rightarrow$ active and $\nu_{e} \rightarrow$ sterile cases are analyzed separately, whereas in the four-neutrino picture they are taken into account simultaneously in a consistent scheme that allows to calculate the allowed regions with the prescription given in Eq. (8). We think that the agreement between the results of the analyses with two and four neutrinos indicate that the physical conclusions are quite robust.

The disappearance of the LMA and LOW regions for

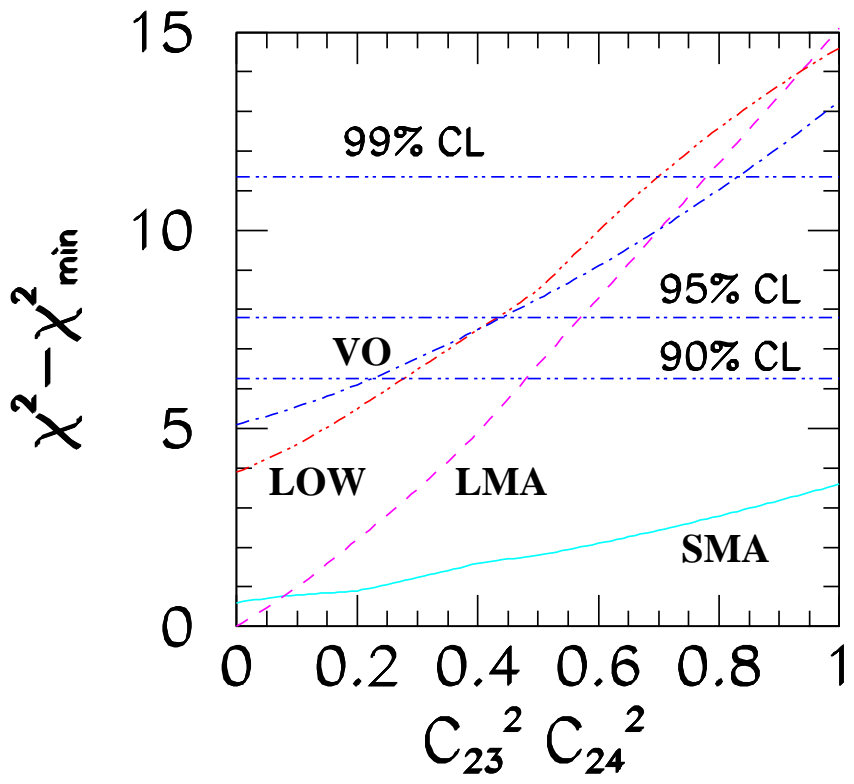

Figure

values of $c_{23}^{2} c_{24}^{2}$ close to one is due to the following reason. Unlike active neutrinos which lead to events in the water Cherenkov detectors by interacting via neutral current with the electrons, sterile neutrinos do not contribute to the Kamiokande and Super-Kamiokande event rates. Therefore a larger survival probability for ${ }^{8} \mathrm{~B}$ neutrinos is needed to accommodate the measured rates. As a consequence a larger contribution from ${ }^{8} \mathrm{~B}$ neutrinos to the Chlorine and Gallium experiments is expected, so that the small measured rate in Chlorine can only be accommodated if less ${ }^{7} \mathrm{Be}$ neutrinos are present in the flux. This is only possible in the SMA solution region, since in the LMA and LOW regions the suppression of ${ }^{7} \mathrm{Be}$ neutrinos is not enough (the survival probability of $\nu_{e}$ 's as a function of neutrino energy is rather flat).

Figure 4 shows that the LMA region at 99\% CL extends to high values of $\Delta m_{21}^{2}$, even above $10^{-3} \mathrm{eV}^{2}$ for $c_{23}^{2} c_{24}^{2} \lesssim 0.1$. Since the atmospheric mass squared difference $\Delta m_{\text {atm }}^{2}$ lies between $10^{-3}$ and $10^{-2} \mathrm{eV}^{2}$ (see $[6]$ ), one may wonder if the solar and atmospheric mass squared differences may coincide and three massive neutrinos may be enough for the explanation of solar, atmospheric and LSND data. The answer to this question is negative, because in the high- $\Delta m_{21}^{2}$ part of the $99 \%$ CL LMA region the mixing angle $\theta_{21}$ is large, $0.3 \lesssim \sin ^{2}\left(\theta_{21}\right) \lesssim 0.7$, and in this case disappearance of $\bar{\nu}_{e}$ 's should be observed in long-baseline reactor experiments, contrary to results of the CHOOZ 23] experiment. In other words, the results of the CHOOZ experiment, that have not been taken into account in the present analysis, forbid the part of the $99 \%$ CL LMA region that extends above $\Delta m_{21}^{2} \simeq 10^{-3} \mathrm{eV}^{2}$. For this reason we cut the plots at this value. 


\section{CONCLUSIONS}

We have considered the two four-neutrino schemes A and $\mathrm{B}$ in Fig. 2 that are compatible with the results of all neutrino oscillation experiments. These two schemes are completely equivalent for neutrino oscillation experiments, but have different phenomenology in $\beta$ and neutrinoless $\beta \beta$ decay experiments (see $[1,24,25])$.

In general the four-neutrino schemes $\mathrm{A}$ and $\mathrm{B}$ allow simultaneous transitions of solar $\nu_{e}$ 's into active $\nu_{\mu}$ 's, $\nu_{\tau}$ 's and sterile $\nu_{s}$, as well as simultaneous transitions of atmospheric $\nu_{\mu}$ 's into active $\nu_{\tau}$ 's and sterile $\nu_{s}$ 's.

We have fitted the data of solar neutrino experiments in terms of neutrino oscillations, that in the schemes under consideration depend on three parameters, $\Delta m_{21}^{2}$, $\tan ^{2} \vartheta_{12}$ and $\cos ^{2} \vartheta_{23} \cos ^{2} \vartheta_{24}$. The allowed regions of these three parameters are depicted in Fig. 4 from which one can see that the SMA region is valid for all values of $\cos ^{2} \vartheta_{23} \cos ^{2} \vartheta_{24}$ (i.e. any combination of $\nu_{e} \rightarrow$ active and $\nu_{e} \rightarrow$ sterile transitions), whereas the LMA, LOW and VO solutions disappear for values of $\cos ^{2} \vartheta_{23} \cos ^{2} \vartheta_{24}$ close to one, where $\nu_{e} \rightarrow \nu_{s}$ transitions are dominant.

Other authors have recently performed fits of the atmospheric neutrino data in the framework of the two fourneutrino schemes A and B [26,27. We expect that in the future a combined fit of solar and atmospheric neutrino data will allow to constraint further the mixing of four neutrinos.

\section{ACKNOWLEDGMENTS}

MCGC and CPG acknowledge partial support by the spanish DGICYT under grants PB98-0693 and PB97-1261, by the Generalitat Valenciana under grant GV99-3-1-01 and by the TMR network grant ERBFMRXCT960090 of the European Union.

[1] S. M. Bilenky, C. Giunti, and W. Grimus, Prog. Part. Nucl. Phys. 43, 1 (1999), arXiv:hep-ph/9812360.

[2] B. T. Cleveland et al., Astrophys. J. 496, 505 (1998).

[3] Kamiokande, Y. Fukuda et al., Phys. Rev. Lett. 77, 1683 (1996).

[4] GAlLEX, W. Hampel et al., Phys. Lett. B447, 127 (1999).

[5] SAGE, J. N. Abdurashitov et al., Phys. Rev. C60, 055801 (1999), arXiv:astro-ph/9907113.

[6] Y. Suzuki (Super-Kamiokande Coll.), Talk presented at the XIX Int. Symposium on Lepton and Photon Interactions at High Energies, Stanford, Aug. 1999 http:// www-sk.icrr.u-tokyo.ac.jp/doc/sk/pub/index.html).

[7] Y. Fukuda et al. (Kamiokande Coll.), Phys. Lett. B 335 , 237 (1994); R. Becker-Szendy et al. (IMB Coll.), Nucl.
Phys. B (Proc. Suppl.) 38, 331 (1995); Y. Fukuda et al. (Super-Kamiokande Coll.), Phys. Rev. Lett. 82, 2644 (1999); W.W.M. Allison et al. (Soudan 2 Coll.), Phys. Lett. B 449, 137 (1999); M. Ambrosio et al. (MACRO Coll.), Phys. Lett. B 434, 451 (1998).

[8] C. Athanassopoulos et al. (LSND Coll.), Phys. Rev. Lett. 81, 1774 (1998);.

[9] C. Giunti, arXiv:hep-ph/9909395.

[10] G.L. Fogli et al., arXiv:hep-ph/9906450.

[11] C. Caso et al., Eur. Phys. J. C3, 1 (1998).

[12] A. Donini, these proceedings.

[13] R.N. Mohapatra, arXiv:hep-ph/9910365; R. Foot, H. Lew and R.R. Volkas, arXiv:hep-ph/0006027.

[14] M. Neubert, these proceedings.

[15] S.M. Bilenky, C. Giunti and W. Grimus, Eur. Phys. J. C 1, 247 (1998), arXiv:hep-ph/9607372; Proc. of Neutrino '96, Helsinki, June 1996, edited by K. Enqvist et al., p. 174, World Scientific, 1997, hep-ph/9609343.

[16] V. Barger, S. Pakvasa, T. J. Weiler, and K. Whisnant, Phys. Rev. D58, 093016 (1998), arXiv:hep-ph/9806328.

[17] S. M. Bilenky, C. Giunti, W. Grimus, and T. Schwetz, Phys. Rev. D60, 073007 (1999), arXiv:hep-ph/9903454.

[18] D. Dooling, C. Giunti, K. Kang, and C. W. Kim, Phys. Rev. D61, 073011 (2000), hep-ph/9908513.

[19] C. Giunti, M. C. Gonzalez-Garcia, and C. Pena-Garay, Phys. Rev. D62, 013005 (2000), arXiv:hep-ph/0001101.

[20] C. Giunti, JHEP 01, 032 (2000), hep-ph/9912211.

[21] J. N. Bahcall, S. Basu, and M. H. Pinsonneault, Phys. Lett. B433, 1 (1998), arXiv:astro-ph/9805135.

[22] M. C. Gonzalez-Garcia, H. Nunokawa, O. L. G. Peres, and J. W. F. Valle, Nucl. Phys. B543, 3 (1999), arXiv:hep-ph/9807305.

[23] M. Apollonio et al. (CHOOZ Coll.), Phys. Lett. B 466, 415 (1999), arXiv:hep-ex/9907037.

[24] C. Giunti, Phys. Rev. D61, 036002 (2000), hep$\mathrm{ph} / 9906275$.

[25] S. M. Bilenky, C. Giunti, W. Grimus, B. Kayser, and S. T. Petcov, Phys. Lett. B465, 193 (1999), arXiv:hep$\mathrm{ph} / 9907234$.

[26] O. Yasuda, these proceedings; arXiv:hep-ph/0006319.

[27] E. Lisi, Talk presented at Neutrino 2000, Sudbury, Canada, June 2000 (http://nu2000.sno.laurentian.ca). 